\title{
Impact of Mechanical Stress on 3-D NAND Flash Current Conduction
}

\author{
Anastasiia Kruv ${ }^{\circledR}$, Antonio Arreghini, Devin Verreck ${ }^{\circledR}$, Mario Gonzalez, \\ Geert Van den bosch, Ingrid De Wolf ${ }^{\circledR}$, Senior Member, IEEE, and Maarten Rosmeulen
}

\begin{abstract}
This work experimentally investigates current conduction in 3-D NAND under up to $7 \mathrm{GPa}$ of externally applied compressive mechanical stress. The impact of channel crystallinity and geometry is studied by comparing four types of Si channels: polycrystalline full channel, single-crystal full channel, polycrystalline macaroni channel, and single-crystal macaroni channel. In the studied channel types, the mechanical stress was found to cause reversible degradation of $I_{O N}$ (up to one order of magnitude) and IOFF (up to seven orders of magnitude). Using TCAD simulations, ION and IOFF deterioration are attributed to carrier mobility and Si bandgap decreases under mechanical stress, respectively. The simulations also suggest that both effects depend on the channel morphology, which results in a different extent of stress impact on the four studied types of Si channels.
\end{abstract}

Index Terms-3-D NAND, current conduction, finite element modeling (FEM), flash, mechanical stress, memory, nanoindentation.

\section{INTRODUCTION}

$\mathbf{S}$ WITCHING from 2-D to 3-D NAND allows to further improve flash memory density and price/bit, while using more relaxed device dimensions and lithography steps. In such 3-D NAND devices, a polycrystalline $\mathrm{Si}$ macaroni channel (MC-POLY) is the industrial standard [1], [2]. This is due to a lower complexity and shorter processing time when compared to the epitaxial growth of single crystalline $\mathrm{Si}$ and the improved gate electrostatic control in comparison with a full-channel geometry. However, MC-POLY devices intrinsically suffer from low ON-current $\left(I_{\mathrm{ON}}\right)$, which further degrades as the channel length increases with the number of stacked memory cells [3]. An additional factor that is expected to negatively impact the $I_{\mathrm{ON}}$ is residual mechanical stress that

Manuscript received July 13, 2020; revised August 28, 2020; accepted September 14, 2020. This work was supported by the imec's Industrial Affiliation Program on Advanced Flash Memory Devices. The review of this article was arranged by Editor P. Narayanan. (Corresponding author: Anastasiia Kruv.)

Anastasiia Kruv and Ingrid De Wolf are with the Department of Materials Engineering, KU Leuven, 3000 Leuven, Belgium, and also with imec, 3001 Leuven, Belgium (e-mail: anastasiia.kruv@imec.be; ingrid.dewolf@imec.be).

Antonio Arreghini, Devin Verreck, Mario Gonzalez, Geert Van den bosch, and Maarten Rosmeulen are with imec, 3001 Leuven, Belgium (e-mail: antonio.arreghini@imec.be; devin.verreck@ imec.be; mario.gonzalez@imec.be; geert.vandenbosch@imec.be; maarten.rosmeulen@imec.be).

Color versions of one or more of the figures in this article are available online at http://ieeexplore.ieee.org.

Digital Object Identifier 10.1109/TED.2020.3024450 inevitably appears during 3-D NAND fabrication [4]. Moreover, mechanical stress is known to enhance junction leakage mechanisms [5] and can thus potentially degrade the 3-D NAND OFF-current $\left(I_{\mathrm{OFF}}\right)$. Besides its negative impact, mechanical stress can be engineered to improve the device performance, as was demonstrated in the strained CMOS technology [5]. However, there is currently a lack of experimental studies that address the impact of mechanical stress on current conduction in industry-relevant 3-D NAND structures.

This work therefore, systematically investigates the impact of externally applied mechanical stress on $I_{\mathrm{ON}}$ and $I_{\mathrm{OFF}}$ of MC-POLY devices. To evaluate the influence of the macaroni geometry and channel polycrystallinity, the MC-POLY structure is compared to three other possible geometries: full-channel polycrystalline Si (FC-POLY), full-channel single crystalline epitaxial-Si (FC-EPI), and macaroni-channel single crystalline epitaxial Si (MC-EPI). A preliminary comparison of the first two geometries was presented by us in [6]. In this work, we complete the study by adding the third geometry (MC-EPI) to the comparison, introducing more accurate calculation of induced mechanical stress, and presenting a theory, supported by TCAD simulations, to explain the observations.

\section{Methodology}

\section{A. Samples}

Schematic cross sections of the four types of studied n-type 3-D NAND SONOS channel architectures are presented in Fig. 1. The fabrication of the devices went as follows. Three pairs of $\mathrm{SiO}_{2} /$ poly-Si $(30 \mathrm{~nm} / 50 \mathrm{~nm})$ bilayers were deposited on a $\mathrm{Si}$ wafer to form the gates and intergate oxides. These layers were then covered with $60 \mathrm{~nm}$ of $\mathrm{SiO}_{2}$, serving as isolation between the top gate and the drain. Next, memory holes with $80 \mathrm{~nm}$ diameter were etched and the oxide $\left(\mathrm{SiO}_{2}\right)$-nitride $\left(\mathrm{Si}_{3} \mathrm{~N}_{4}\right)$-oxide $\left(\mathrm{SiO}_{2}\right), \mathrm{ONO}$, gate-stack $(7 \mathrm{~nm}$ $\mathrm{SiO}_{2} / 6 \mathrm{~nm} \mathrm{Si} \mathrm{N}_{4} / 5 \mathrm{~nm} \mathrm{SiO}_{2}$ ) was deposited in the memory hole.

To fabricate FC-POLY, a low pressure (LP)-CVD deposition of amorphous Si was used to fill the memory hole and to form a $180 \mathrm{~nm}$ thick drain. Then, Si was crystallized by $2 \mathrm{~h}$ furnace anneal at $650{ }^{\circ} \mathrm{C}$ [7]. In the case of FC-EPI, a single-crystalline channel was epitaxially grown with $\mathrm{CVD}$ at $810{ }^{\circ} \mathrm{C}$ and a 30-nm Ti/TiN metal drain was deposited [8]. To fabricate MC-POLY, an 11-nm-thick amorphous Si liner was deposited in the memory hole, followed by filling the rest of the memory hole with an $\mathrm{SiO}_{2}$ filler. After that, the channel was 


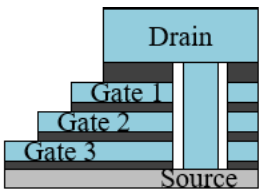

(a)

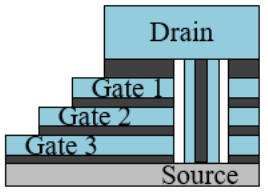

(c)

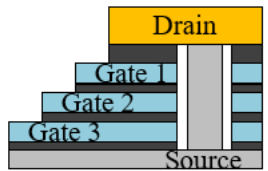

(b)

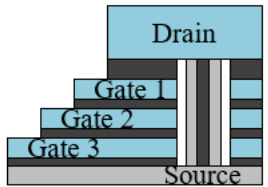

(d)

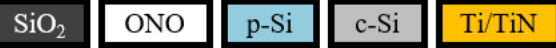

Fig. 1. Schematic illustration of the four investigated channel types. (a) Full channel polycrystalline Si. (b) Full channel single-crystalline Si. (c) Macaroni channel polycrystalline Si. (d) Macaroni channel singlecrystalline Si.

crystallized by high-temperature annealing $\left(1050{ }^{\circ} \mathrm{C}, 45 \mathrm{~s}\right)$ and the 180-nm-thick polysilicon drain was deposited [3]. The processing of MC-EPI initially followed the same path as the MC-POLY. However, after the filler formation, the amorphous $\mathrm{Si}$ liner was recessed and replaced by epitaxially grown $\mathrm{Si}$. Following that, the drain was formed in the same way as in the case of MC-POLY [9]. After the channel and drain formation, all four types of the devices went through staircase patterning and contact formation.

\section{B. Mechanical Stress Application}

Mechanical stress was induced in the sample by applying a vertical mechanical force on top of the device. This was done using a Hysitron TI 950 nanoindenter, equipped with a cube-corner diamond tip. Several values of mechanical force, ranging from 0.2 to $3.5 \mathrm{mN}$, were applied, separated by time intervals where the force was removed. The device was electrically characterized before, during and after each force application (see Section II-D). This allowed to record the initial state of the device, the impact of the stress, and the device recovery after stressing. During the application of the mechanical stress, the force experienced by the tip was recorded as a function of the tip vertical displacement.

To precisely apply the force above the channel, the drain topography was scanned with the tip [Fig. 2(a)]. This allowed to place the tip in the center of the square drain region where the channel is located by design, as illustrated in Fig. 2(a). The topography scan after the force application permitted to determine the actual indentation location. Because of the environmental noise, a small positioning offset $(<350 \mathrm{~nm})$ was observed. Since the offset impacts the value of mechanical stress induced in the channel, it was included in the mechanical stress calculation.

The experiments investigate the impact of up to $7 \mathrm{GPa}$ compressive stress. The stress is limited to compressive because a nanoindenter cannot apply vertical tensile stress. Although the gigapascal range exceeds the typical values of residual stress ( 350 MPa [4], [10]), its exploration is interesting for

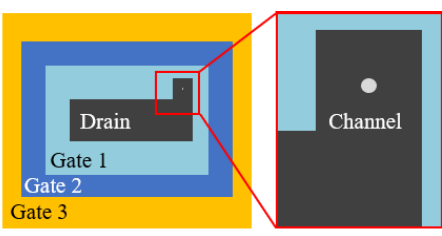

(a)

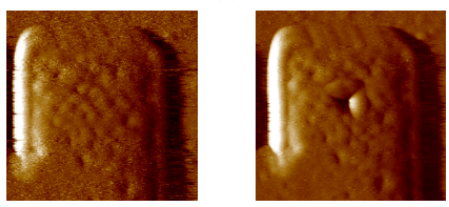

(b)

(c)

Fig. 2. (a) Device top-down layout indicates the position of the channel. A topography scan before (b) and after (c) force application illustrates the capability of applying the force above the channel.

TABLE I

Material Properties USEd IN THE FEM

\begin{tabular}{|c|c|}
\hline Parameter & Value \\
\hline Si Young's Modulus (YM) & $169 \mathrm{GPa}$ \\
\hline $\mathrm{SiO}_{2}$ YM & $73 \mathrm{GPa}$ \\
\hline $\mathrm{Si}_{3} \mathrm{~N}_{4}$ YM & $256 \mathrm{GPa}$ \\
\hline $\mathrm{Si}$ Poisson ratio (PR) & 0.22 \\
\hline $\mathrm{SiO}_{2}$ PR & 0.22 \\
\hline $\mathrm{Si}_{3} \mathrm{~N}_{4}$ PR & 0.28 \\
\hline $\mathrm{SiO}_{2}$ yield stress & $5.5 \mathrm{GPa}$ \\
\hline
\end{tabular}

better understanding the physics and providing insights for future stress engineering, which, based on strained CMOS experience, can be expected to produce gigapascal stress [11].

\section{Mechanical Stress Calculation}

As the induced mechanical stress could not be measured in situ, its value was calculated using finite element modeling (FEM). The induced stress depends on the applied force, indentation offset, tip geometry, device structure, and materials. All these parameters were accounted for in a halfsymmetry 3-D model in MSC.Marc software [12], illustrated in Fig. 3(a). The cube-corner tip used in the experiment was modeled by an equivalent conical tip with a $400-\mathrm{nm}$ diameter. The geometrical parameters of the equivalent tip were extracted from the calibration, which methodology is discussed in [13]. All materials were modeled as isotropic linear elastic except for the topmost $\mathrm{SiO}_{2}$ layer. This layer was treated as isotropic elastoplastic to account for the plastic deformation occurring in it due to the direct contact with the nanoindenter (Fig. 3). The material parameters used in the modeling are summarized in Table I.

An example of force to mechanical stress conversion is presented in Fig. 4. A good match of the simulated and experimental force-displacement curves is obtained, giving confidence in the simulated mechanical stress values [Fig. 4(a)]. As can be seen in Fig. 4(b), the application of a vertical force to the sample produces triaxial mechanical stress. Since the compressive mechanical stress along the channel (stress Z) is significantly larger than the two other components, the electrical performance will be reported in the following sections as a function of it. Because this stress component has a 

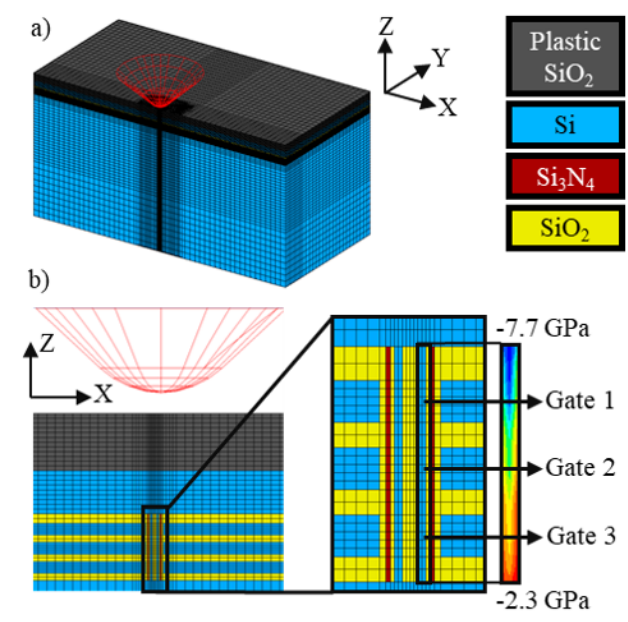

Fig. 3. (a) Half-symmetry 3-D FEM model ( $Z X$ is the plane of symmetry) used for mechanical stress calculation. (b) Stress in the channel is extracted for each cell at the position in the $Z$-direction corresponding to the middle of the cell's gate.

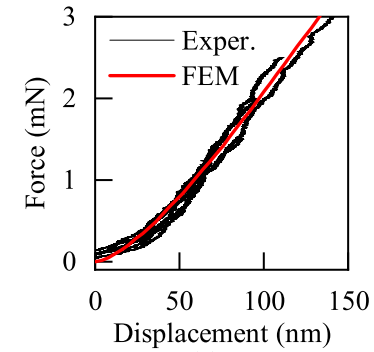

(a)

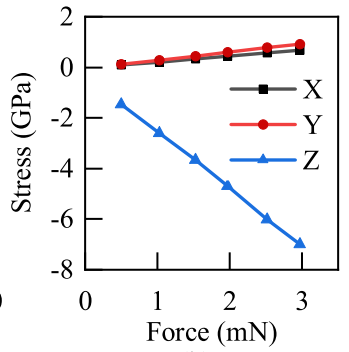

(b)
Fig. 4. (a) Comparison of the six experimental force-displacement curves with the simulated one shows their good match and indicates that the modeling is representative. (b) Simulation indicates that stress in the channel increases with the applied force, and that the vertical stress (Z) dominates the two other components. The presented stress values are average values extracted for the case of the MC-EPI top cell with a $30 \mathrm{~nm}$ tip offset.

gradient along the channel [Fig. 3(b)], the mechanical stress was extracted individually for each of three memory cells at the points indicated in Fig. 3(b).

\section{Electrical Characterization Under Mechanical Stress}

As the nanoidenter does not have functionality for electrical characterization, miniature electrical probes had to be placed in its chamber to enable in situ characterization. The electrical performance of the devices was evaluated by measuring their transfer characteristics at different values of applied mechanical force (stress) using Keithley K2600 source-measure units and four electrical probes from Imina Technology. Transfer characteristics of each of the three memory cells present in each device (Fig. 1) were consecutively measured, while keeping the two other cells in the pass mode. The drain current is thereby limited by the cell being measured, which allows the change in $I_{\mathrm{D}} V_{\mathrm{G}}$ to be associated with the mechanical stress induced in this cell. In the measurements, the source was grounded via the substrate and the drain, and pass-mode gates were kept at 1 and $7 \mathrm{~V}$, respectively. In all cases, the gate leakage was negligible.

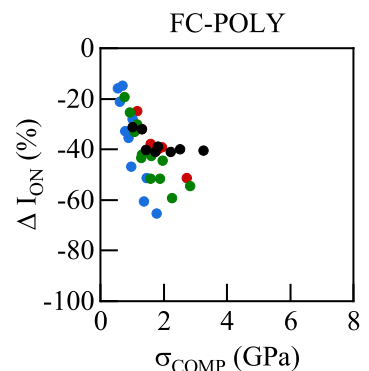

(a)

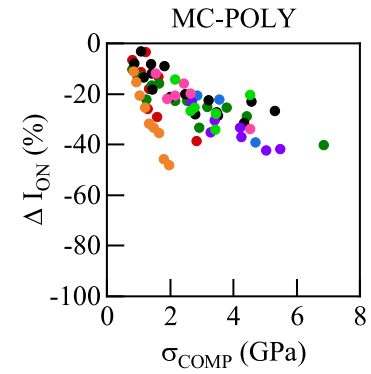

(c)

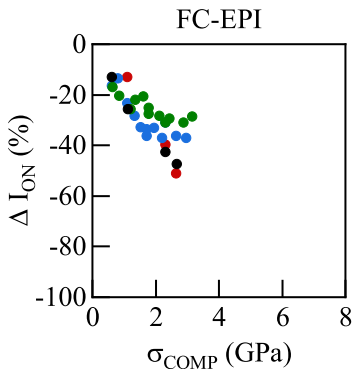

(b)

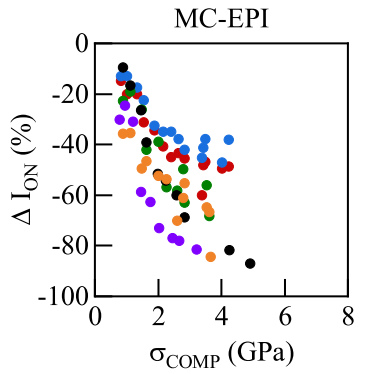

(d)
Fig. 5. Measured ION change under vertical compressive mechanical stress for four studied channel geometries: (a) FC-POLY, (b) FC-EPI, (c) MC-POLY, and (d) MC-EPI. The ION degradation extent depends on the channel type. Within each channel type, the color indicates different devices.

The $I_{\mathrm{ON}}$ and $I_{\mathrm{OFF}}$ were obtained from the transfer characteristics for different values of applied mechanical stress. The $I_{\mathrm{OFF}}$ was defined as the minimum drain current, while the $I_{\mathrm{ON}}$ was extracted as the $I_{\mathrm{D}}$ at $2 \mathrm{~V}$ overdrive relative to the threshold voltage defined at $I_{\mathrm{D}}=10 \mathrm{nA}$. The stressinduced change in $I_{\mathrm{ON}}$ and $I_{\mathrm{OFF}}$ was calculated relative to the values before any stress application. Due to time-consuming tip positioning and manual electrical probing, a rather limited number of devices of each channel type could be measured.

To exclude the impact of stress-induced defects, only data points before a breaking point were analyzed, except for one case, explicitly indicated in a latter section. The device recovery was judged by the overlap of the transfer characteristics recorded after each force application with the one measured before any mechanical stressing.

\section{Results And Discussion}

\section{A. Impact of Mechanical Stress on ION}

Compressive mechanical stress degrades the $I_{\mathrm{ON}}$ in all studied channel types (Fig. 5), which we attribute to the stress-induced electron mobility deterioration [14]. When the mechanical stress increases, $\Delta I_{\mathrm{ON}}$ first follows a linear trend and then saturates for some devices. The maximum degradation of $87 \%$ is found for MC-EPI at $5 \mathrm{GPa}$. Fig. 6 presents the distribution of $I_{\mathrm{ON}}$ stress sensitivity $(\delta)$ for the studied channel types. The sensitivity was calculated for each device as a slope of a fit to data points (indicated in Fig. 5) in the linear regime. Although there is a spread in $\delta$ between the devices, in all cases the average $\delta$ does not exceed 3\%/100 MPa. Since the residual mechanical stress in the 3-D NAND channel is estimated to be $\sim 350 \mathrm{MPa}$ in the industrial devices [4], [10], an $I_{\mathrm{ON}}$ degradation of around $10 \%$ is expected under compressive stress. At the same time, as the compressive and tensile 


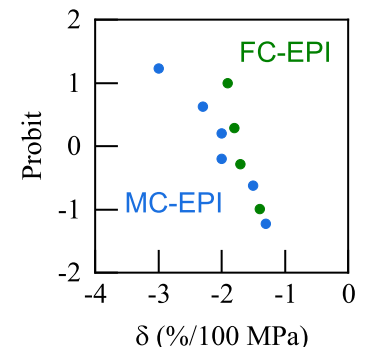

(a)

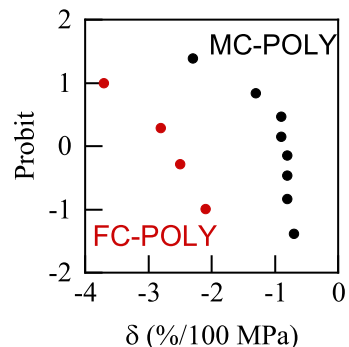

(b)
Fig. 6. Measured ION stress sensitivity ( $\delta$ ) for (a) EPI and (b) POLY devices. The sensitivity is similar for the EPI devices and different for the POLY samples.

stress are known to have a symmetric impact on mobility [14], the $I_{\mathrm{ON}}$ might be expected to improve by a similar percentage under tensile stress.

We now investigate the impact of channel geometry and morphology on the $I_{\mathrm{ON}}$ stress sensitivity. A comparison of the $\delta$ of FC-EPI and MC-EPI channels reveals that their mean sensitivity is not significantly different [Fig. 6(a)]. This can be caused by equivalent crystallographic orientations of the two channels, as the stress impact on mobility is known to be orientation-dependent [14]. It also suggests that an extra interface and a reduced channel volume caused by the filler oxide do not impact the sensitivity of the single crystalline samples. Fig. 6(b) shows that in contrast to the EPI devices, the mean sensitivity is lower in MC-POLY when compared to FC-POLY. Given the fact that the channel geometry itself does not cause significant differences in EPI samples, we argue that the difference between the POLY devices can be attributed to the impact of the channel geometry on the Si morphology (e.g., grain size and orientation, as well as grain boundary defectivity).

To corroborate this hypothesis, $\delta$ was qualitatively simulated with the Minimos simulator from Global TCAD Solutions [15] for the FC device geometry. The impact of the mechanical stress on the carrier mobility (and thus on $\delta$ ) was incorporated by activating a first-order orientation-dependent piezoresistive model [16]. The effect of the grain boundary defectivity was accounted for by changing the carrier velocity at the boundaries, since larger defectivity translates into a lower carrier velocity via enhanced scattering [17]. For each combination of the average grain size and carrier velocity, the simulations were performed for 30 random combinations of differently oriented grains that compose the channel.

The simulation results confirm the impact of grain size, orientation, and channel defectivity on $\delta$. Fig. 7(a) indicates that for each average grain size, different combinations of grain orientations result in various $\delta$. When the average grain size increases, and thus number of grain boundaries reduces, the sensitivity spread increases, and higher values of $\delta$ are obtained. The same trend in $\delta$ is observed when the carrier velocity at the grain boundaries increases, as depicted in Fig. 7(b). This can be explained by the carrier transport becoming determined by the stress-dependent mobility inside the grains, rather than by stress-independent conduction at the grain boundaries. Thus, depending on the exact grain configuration and relative dominance of conduction inside

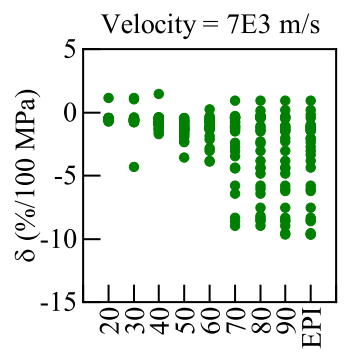

Average grain size (nm)

(a)

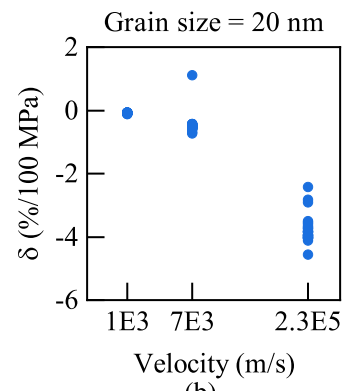

(b)
Fig. 7. Simulated ION stress sensitivity as a function of (a) average grain size and (b) carrier velocity at the grain boundaries. For each parameter value, 30 channels with random grain orientations are presented. The simulations indicate that the sensitivity magnitude and spread depend on the channel morphology.

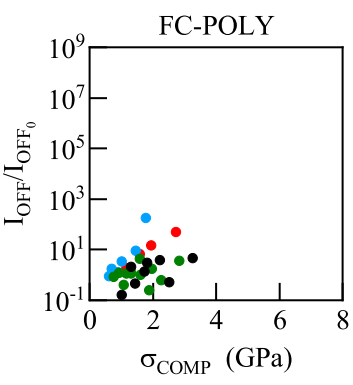

(a)

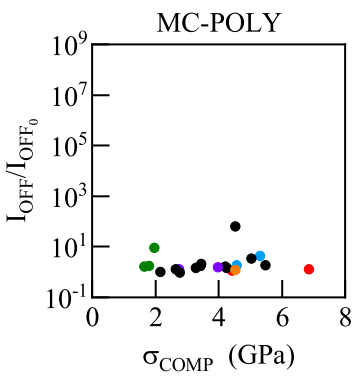

(c)

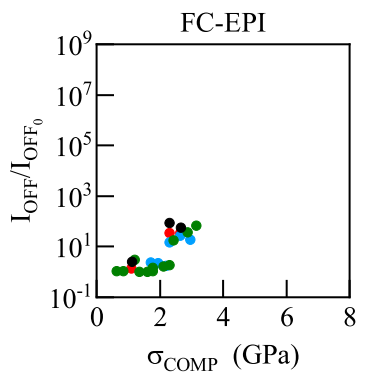

(b)

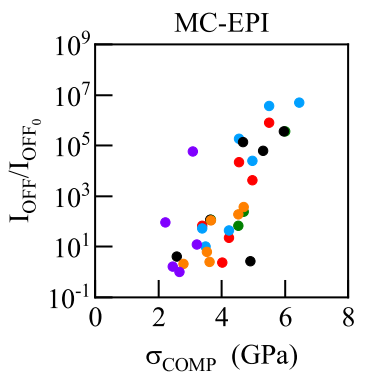

(d)
Fig. 8. Experimentally observed /OFF change under vertical compressive mechanical stress for four studied channel geometries: (a) FC-POLY, (b) FC-EPI, (c) MC-POLY, and (d) MC-EPI. The IOFF deterioration extent depends on the channel architecture. Within each channel type, the color represents different devices.

the grains and across the grain boundaries, different $I_{\mathrm{ON}}$ stress sensitivity can be obtained. We therefore, attribute the difference between the poly configurations in Fig. 6(b) to the difference in the morphology of their channels.

\section{B. Impact of Mechanical Stress on I OFF}

The drain-to-source leakage current of all studied devices degrades under the application of compressive mechanical stress (Fig. 8). A deterioration of up to two orders of magnitude at $3 \mathrm{GPa}$ is observed for FC devices. For MC-EPI, the leakage increase can be up to a remarkable seven orders of magnitude at $5.5 \mathrm{GPa}$. This stress level results in flat transfer characteristics of MC-EPI, which, nevertheless, return to their original shape when mechanical stress is removed, as shown in Fig. 9(d). However, when similar mechanical stress (5.8 GPa) is applied to MC-POLY, the reversible leakage increase is very limited [Fig. 9(c)]. Flat-transfer characteristics are observed 


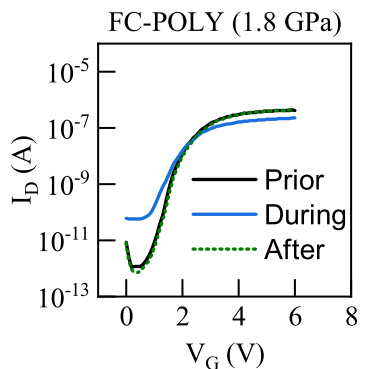

(a)

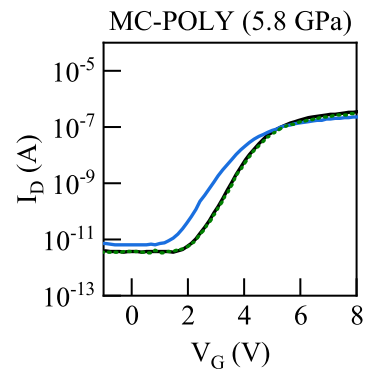

(c)

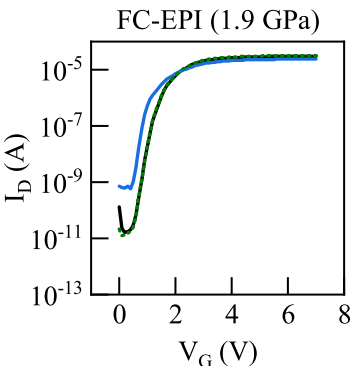

(b)

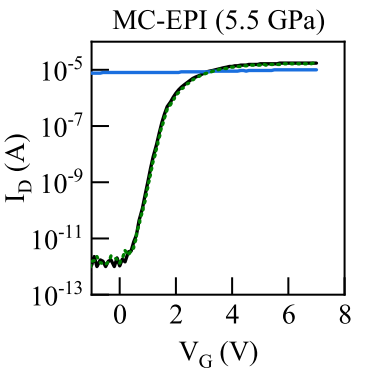

(d)
Fig. 9. Typical top cell $I_{D} V_{G}$ curves measured prior, during, and after stress application. (a) FC-POLY (1.8 GPa). (b) FC-EPI (1.9 GPa). (c) MC-POLY (5.8 GPa). (d) MC-EPI (5.5 GPa). Overlap of the "prior" and "after" characteristics indicates the device recovery, which is observed even after seven orders of magnitude IOFF increase in MC-EPI.

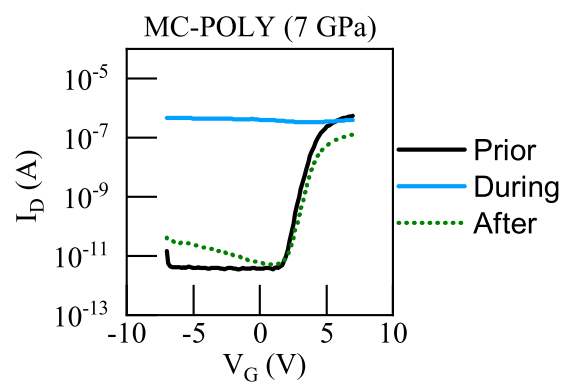

Fig. 10. MC-POLY top cell-transfer characteristics which shows that significant loFF increase is possible for this architecture, but happens under high stress from which the device does not recover.

only for MC-POLY subjected to $7 \mathrm{GPa}$ mechanical stress, from which the device does not recover (Fig. 10). It should be noted, that in the case of the MC devices, the minimum measured current is limited by the tool resolution. Consequently, it is plausible that the calculated $I_{\mathrm{OFF}}$ increase under mechanical stress is underestimated for these channel structures.

We attribute the observed trends to the Si bandgap narrowing under mechanical stress [5]. To support this hypothesis, the transfer characteristics of a device with FC-EPI geometry were qualitatively simulated in Synopsys Sentaurus Device [18]. In the simulation, the current conduction was governed by the drift-diffusion equation. The impact of mechanical stress was accounted for by simulating the transfer characteristics for different values of $\mathrm{Si}$ bandgap, which is known to decrease under mechanical stress [5]. The simulation results (Fig. 11) indeed indicate that when the bandgap decreases, the OFF-current increases by orders of magnitude and becomes comparable to $I_{\mathrm{ON}}$ at a bandgap of $\sim 0.2 \mathrm{eV}$. Comparison of the band diagrams at 1.124 and $0.2 \mathrm{eV}$

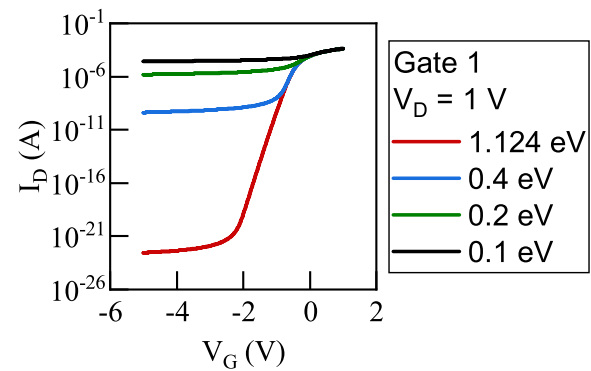

Fig. 11. Simulated $I_{D} V_{G}$ curves of 3-D NAND top cell illustrate the IOFF increase with decreasing Si bandgap.

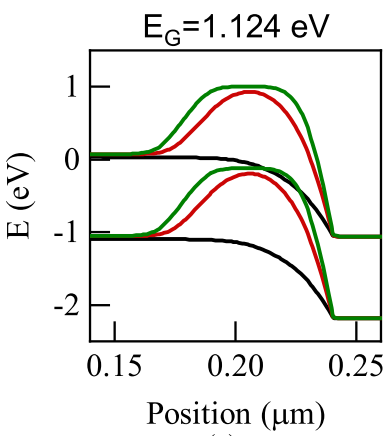

(a)

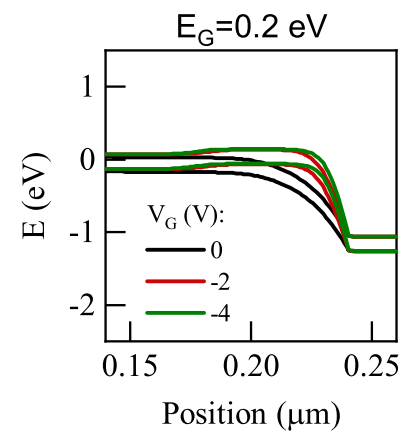

(b)
Fig. 12. Simulated band diagrams demonstrate that the gate is unable to significantly increase the energy barrier in the top cell channel when the bandgap is reduced from (a) $1.124 \mathrm{eV}$ to (b) $0.2 \mathrm{eV}$.

(Fig. 12) reveals that in the latter case the gate is unable to significantly increase the energy barrier between the source and drain and thus to switch off the current. This happens due to the surface potential pinning at high carrier concentration, which makes it almost independent of the gate bias. The carrier concentration required for pinning is achieved at more positive gate bias for a lower bandgap.

A significant bandgap decrease to the values indicated by the simulations can indeed be expected in the conducted experiments. As reported in Fig. 8, the induced mechanical stress can reach $7 \mathrm{GPa}$ in MC-EPI. At this stress level, the bandgap of [001] $\mathrm{Si}$ is estimated to be approximately $0.4 \mathrm{eV}$ based on the deformation potential model in Sentaurus Device [18], as indicated in Fig. 13. Keeping in mind the qualitative nature of the TCAD simulations, this value is considered to be sufficiently close to $0.2 \mathrm{eV}$ at which an almost flat $I_{\mathrm{D}} V_{\mathrm{G}}$ is observed in the simulations.

The difference in $I_{\mathrm{OFF}}$ increase between various channels can be attributed to the orientation-dependent bandgap stress sensitivity (Fig. 13). The high sensitivity of the [001] orientation can explain a significant leakage increase in MC-EPI. At the same time, comprising grains with lower sensitivity can make MC-POLY less sensitive. Thus, at similar stress values, the bandgap in the channel of MC-POLY can be larger than that of MC-EPI, leading to a smaller $I_{\mathrm{OFF}}$ increase. However, if stress is increased to the value that causes a sufficiently small bandgap, the device shows an $I_{\mathrm{OFF}}$ increase but does not recover after stress application, (Fig. 10). The assumption that MC-POLY comprises grains with lower stress sensitivity is also in agreement with its low $I_{\mathrm{ON}}$ stress sensitivity, which is also orientation-dependent. 


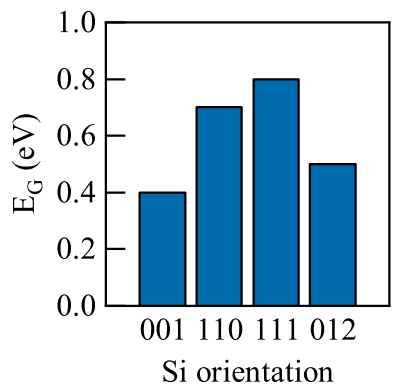

Fig. 13. Simulations at 7-GPa compressive stress indicate that the $\mathrm{Si}$ bandgap stress sensitivity depends on the Si crystallographic orientation.

As the tensile mechanical stress also results in a Si bandgap decrease [19], its presence in the structures can be expected to degrade the $I_{\mathrm{OFF}}$. As the Si bandgap sensitivity to tensile stress is 1.6 times lower than to compressive stress [19], and given the $I_{\mathrm{OFF}}$ dependence on the bandgap (Fig. 11), the $I_{\mathrm{OFF}}$ increase at a given tensile stress is expected to be a few orders of magnitude lower than at the same value of compressive stress.

\section{CONCLUSION}

This work systematically investigated the impact of vertical compressive mechanical stress ON-current conduction in four types of 3-D NAND channels: full channel poly-Si, full channel epitaxial-Si, macaroni channel poly-Si, and macaroni channel epitaxial-Si. In all studied devices reversible degradation of $I_{\mathrm{ON}}$ and $I_{\mathrm{OFF}}$ was observed, which was attributed to a decrease in mobility and bandgap under mechanical stress, respectively. The average $I_{\mathrm{ON}}$ stress sensitivity was measured to be below $3 \% / 100 \mathrm{MPa}$, while up to seven orders of magnitude $I_{\mathrm{OFF}}$ increase was recorded at 5.5-GPa mechanical stress. The simulation suggests that the extent of $I_{\mathrm{ON}}$ and $I_{\mathrm{OFF}}$ degradation under stress is impacted by channel morphology.

This study indicates that residual compressive mechanical stress must be limited during 3-D NAND fabrication, to prevent it from negatively impacting the current conduction. At the same time, tensile stress engineering might be considered in future studies to improve the $I_{\mathrm{ON}}$. However, since tensile mechanical stress results in the Si bandgap decrease [19], its introduction can be expected to degrade the $I_{\mathrm{OFF}}$.

\section{ACKNOWLEDGMENT}

The authors acknowledge the contributions of G. Hiblot, D. Waldhoer (TU Wien), and Z. Stanojević (Global TCAD Solutions) to this work.

\section{REFERENCES}

[1] Y. Fukuzumi et al., "Optimal integration and characteristics of vertical array devices for ultra-high density, bit-cost scalable flash memory," in IEDM Tech. Dig., Dec. 2007, pp. 449-452.

[2] A. S. Spinelli, C. M. Compagnoni, and A. L. Lacaita, "Variability effects in nanowire and macaroni MOSFETs-Part I: Random dopant fluctuations," IEEE Trans. Electron Devices, vol. 67, no. 4, pp. 1485-1491, Apr. 2020.

[3] G. Congedo et al., "Analysis of performance/variability trade-off in macaroni-type 3-D NAND memory," in Proc. IEEE 6th Int. Memory Workshop (IMW), May 2014, pp. 1-4.

[4] Y. Namkoong, H. J. Yang, and Y. H. Song, "Mechanical stress distribution and the effects of process parameter changes in vertical NAND flash memory," J. Nanosci. Nanotechnol., vol. 17, no. 7, pp. 5055-5060, Jul. 2017.

[5] Y. Sun, S. E. Thompson, and T. Nishida, "Physics of strain effects in semiconductors and metal-oxide-semiconductor field-effect transistors," J. Appl. Phys., vol. 101, no. 10, May 2007, Art. no. 104503.

[6] A. Kruv et al., "Impact of mechanical stress on the electrical performance of 3D NAND," in Proc. IEEE Int. Rel. Phys. Symp. (IRPS), Mar. 2019, pp. 1-5.

[7] G. Van Den Bosch et al., "Highly scaled vertical cylindrical SONOS cell with bilayer polysilicon channel for 3-D NAND flash memory," IEEE Electron Device Lett., vol. 32, no. 11, pp. 1501-1503, Nov. 2011.

[8] E. Capogreco et al., "Integration and electrical evaluation of epitaxially grown $\mathrm{Si}$ and SiGe channels for vertical NAND memory applications," in Proc. IEEE Int. Memory Workshop (IMW), May 2015, pp. 1-4.

[9] R. Delhougne et al., "First demonstration of monocrystalline silicon macaroni channel for 3-D NAND memory devices," in Proc. IEEE Symp. VLSI Technol., Jun. 2018, pp. 203-204.

[10] V. Cherman et al., "Evaluation of mechanical stress induced during IC packaging," in Proc. IEEE 68th Electron. Compon. Technol. Conf. (ECTC), May 2018, pp. 2168-2173.

[11] L. Smith et al., "Exploring the limits of stress-enhanced hole mobility," IEEE Electron Device Lett., vol. 26, no. 9, pp. 652-654, Sep. 2005.

[12] Finite Element Modeling Software Used in This Work. Accessed: Sep. 19, 2020. [Online]. Available: http://www.mscsoftware.com/product/marc

[13] O. O. Okudur, K. Vanstreels, I. De Wolf, and U. Hangen, "Extraction of elastic modulus of porous ultra-thin low-k films by two-dimensional finite-element simulations of nanoindentation," J. Appl. Phys., vol. 119, no. 2, Jan. 2016, Art. no. 025302.

[14] Y. Kanda, "A graphical representation of the piezoresistance coefficients in silicon," IEEE Trans. Electron Devices, vol. 29, no. 1, pp. 64-70, Jan. 1982.

[15] Global TCAD Solutions. Accessed: Sep. 19, 2020. [Online]. Available: http://www.globaltcad.com

[16] Minimos-NT User Manual, Global TCAD Solutions GmbH, Vienna, Austria, Sep. 2017, pp. 197-202.

[17] D. Verreck et al., "3D TCAD model for poly-Si channel current and variability in vertical NAND flash memory," in Proc. Int. Conf. Simul. Semiconductor Processes Devices (SISPAD), Sep. 2019, pp. 1-4.

[18] Sentaurus Device User Guide, Synopsys Corporate Headquarters, Mountain View, CA, USA, Jun. 2018.

[19] T.-K. Kang, "Evidence for silicon bandgap narrowing in uniaxially strained MOSFETs subjected to tensile and compressive stress," IEEE Electron Device Lett., vol. 33, no. 6, pp. 770-772, Jun. 2012. 